\title{
Designing Shapelets for Interpretable Data-Agnostic Classification
}

\author{
Riccardo Guidotti \\ University of Pisa \\ Pisa, Italy \\ riccardo.guidotti@unipi.it
}

\author{
Anna Monreale \\ University of Pisa \\ Pisa, Italy \\ anna.monreale@unipi.it
}

\begin{abstract}
Time series shapelets are discriminatory subsequences which are representative of a class, and their similarity to a time series can be used for successfully tackling the time series classification problem. The literature shows that Artificial Intelligence (AI) systems adopting classification models based on time series shapelets can be interpretable, more accurate, and significantly fast. Thus, in order to design a data-agnostic and interpretable classification approach, in this paper we first extend the notion of shapelets to different types of data, i.e., images, tabular and textual data. Then, based on this extended notion of shapelets we propose an interpretable data-agnostic classification method. Since the shapelets discovery can be time consuming, especially for data types more complex than time series, we exploit a notion of prototypes for finding candidate shapelets, and reducing both the time required to find a solution and the variance of shapelets. A wide experimentation on datasets of different types shows that the data-agnostic prototypebased shapelets returned by the proposed method empower an interpretable classification which is also fast, accurate, and stable. In addition, we show and we prove that shapelets can be at the basis of explainable AI methods.
\end{abstract}

\section{CCS CONCEPTS}

- Computing methodologies $\rightarrow$ Supervised learning; Knowledge representation and reasoning; Instance-based learning; Classification and regression trees; $\bullet$ Information systems $\rightarrow$ Decision support systems; Nearest-neighbor search.

\section{KEYWORDS}

Interpretable Machine Learning, Explainable Artificial Intelligence, Transparent Classification Model, Decision Support Systems

\section{ACM Reference Format:}

Riccardo Guidotti and Anna Monreale. 2021. Designing Shapelets for Interpretable Data-Agnostic Classification. In Proceedings of the $2021 \mathrm{AAAI} / \mathrm{ACM}$ Conference on AI, Ethics, and Society (AIES '21), May 19-21, 2021, Virtual Event, USA. ACM, New York, NY, USA, 11 pages. https://doi.org/10.1145/ 3461702.3462553

Permission to make digital or hard copies of all or part of this work for personal or classroom use is granted without fee provided that copies are not made or distributed for profit or commercial advantage and that copies bear this notice and the full citation on the first page. Copyrights for components of this work owned by others than ACM must be honored. Abstracting with credit is permitted. To copy otherwise, or republish, to post on servers or to redistribute to lists, requires prior specific permission and/or a fee. Request permissions from permissions@acm.org.

AIES '21, May 19-21, 2021, Virtual Event, USA

(C) 2021 Association for Computing Machinery.

ACM ISBN 978-1-4503-8473-5/21/05 . .\$15.00

https://doi.org/10.1145/3461702.3462553

\section{INTRODUCTION}

Explainable Artificial Intelligence (AI) is a recent flourishing research activity largely debated nowadays [10]. This is mainly due to the wide diffusion of machine learning-based AI systems in a plethora of applications such as credit score, insurance risk, healthcare, IoT, in which both the predictive accuracy and the comprehensibility of the system reasoning are of the utmost importance [28]. Interpretability is a crucial building block of a trust-worthy interaction between humans and AI systems, especially in high-stakes decision making, such as clinical diagnosis, because it enables users to take conscious and accountable decisions [24]. Also, we aspire for user acceptance of the machine decision and of its way of reasoning. If the machine logic is transparent and accessible, as humans, we tend to trust more a decision process using a logic similar to that one of a human being, rather than a reasoning that we can understand but that is outside the human way of thinking [26]. For instance, we recognize a cat in an image by the presence of a tail, pointed ears, and mustaches, not from pixels having certain values.

This kind of reasoning is based on important sub-parts of the instance processed by an AI system. In time series analysis, different works have proposed approaches $[19,39,40]$ leading to an interpretable classification based on a data representation exploiting shapelets. Shapelets are time series subsequences particularly suitable for separating instances among different classes. Indeed, shapelets capture the most informative portion of data contributing to the final prediction. This property makes shapelets interpretable since they provide users with the reason for a specific prediction. In addition, shapelets are subsequences, i.e., parts of the original instance that humans can easily accept as concepts used for classification. To the best of our knowledge, research on shapelets is focused on time series and on speeding up their discovery [18, 22, 30].

We propose a Data-Agnostic classification method via sHapelets (DASH), that is based on the extension of the notion of shapelets to different types of data: image, tabular, and textual data. DASH, first efficiently extracts shapelets exploiting data prototypes obtained by a clustering process. Then, it exploits these shapelets to transform the training data into a representation expressing the distance of the instances from the shapelets. Finally, it trains an interpretable classifier such as a decision tree, nearest neighbor or logistic regression [16] on the dataset transformed into distances. Such an interpretable classifier can be used as transparent model for an AI system for which there is a requirement of explainability. As an alternative, DASH can be exploited for extending existing explanation procedures such as LIME [33], TREPAN [7] or LORE [15] such that the features importance or the rules returned as explanations refers to shapelets instead of individual variables, pixels, or words. Indeed, 
given any type of data, shapelets provide a comprehensible intuition about the informative portion of the instance that determines the class label.

A wide experimentation on different type of data and datasets with respect to various interpretable classifiers shows that, DASH reaches performance comparable to the state-of-the-art in terms of accuracy. Moreover, DAsH reduces both the computational time required for the shapelets extraction and the variance among shapelets obtained from different executions, which is a typical drawback of efficient shapelet discovery approaches based on random-shapelets [19]. Besides, we show how DAsH can extend existing explanation methods and improve their performance and their interpretability.

The rest of this paper is organized as follows. First, we discuss related works and we formally recall the basic notions of shapelet discovery. Then, we formalize the shapelets for images, tabular and textual data, and we illustrate the proposed methodology. After that we present qualitative and quantitative experimental results. Finally, we summarize our contribution, the limitations of the proposed methodology, and future research directions.

\section{RELATED WORK}

The notion of shapelets is mainly related to time series. The definition of time series shapelets and their usage for classification is initially proposed in [39]. The main drawback of this method is its large computational complexity. As a consequence, shapelet research has been mainly focused on the design of approaches to make more efficient the shapelet search. The proposal in [25] improves [39] by using a cache for faster distance computation. In [22] the shapelet discovery is extended for extracting multiple shapelets to increase the predictive power for multi-class problems. The procedure transform a time series into a feature vector containing the distances between the time series and the best shapelets, and then uses simple classifiers trained on such features vectors to perform the classification. An extension has been proposed in [18].

A strategy widely adopted to address the problem of time complexity is based on random sampling of candidate shapelets [12, 19, $20,32,37]$. In [13, 14], shapelets are learned by a gradient-descentbased optimization algorithm that introduces a random effect mitigated by an evolutionary computation. Another line of research tries to reduce the dimensionality of each time series by approximating them in a compact representation. In [30] it is exploited a symbolic representation of the time series, called SAX [21], and a random projection on the SAX representation to reduce the computational complexity. In [9] it is introduced an approach for learning transform-invariant representative prototypes of time series subsequences. Then, each time series can be approximated by combining those prototypes and then, it can be easily classified. Finally, [36] combines a CNN to classify time series with an adversarial technique to regularize the parameters of the network to learn shapelets.

To the best of our knowledge, shapelets have not been previously used for classification of a type of data different from time series. However, the notion of shapelets has been already used for image analysis. In [31] it is presented a method for image analysis based on a linear decomposition of each object into shapelets representing a collection of compact disjoint objects of arbitrary shapes. In $[34,38]$ it is proposed a learning algorithm for shapelet features able to discriminate between pedestrians and non-pedestrians.

Our work differs from those in literature in three aspects. First, the extension of the shapelet notion to different data types (image, text and tabular data) enabling a distance-based data transformation making the interpretable classifiers data independent. Second, the usage of a prototype-based approach for speeding up the shapelets extraction process. Third, the usage of shapelets for explanation.

\section{SETTING THE STAGE}

We recall the notion of time series shapelet useful for understanding our proposal. A shapelet is informally defined as the most informative (representative) time series subsequence of a class. The idea of shapelet discovery presented in [39] is based on the information gain and follows a logic similar to that one of the node selection in a decision tree [29].

We formally define a time series as:

Definition 3.1 (Time Series). A time series is an ordered set of $m$ real-valued variables $x=\left\{t_{1}, \ldots, t_{m}\right\}$, i.e., $t_{i} \in \mathbb{R}$.

Data points $t_{i}$ are temporally ordered at equal time intervals. A subsequence is a local subsection of a time series.

Definition 3.2 (Subsequence). Given a time series $x \in \mathbb{R}^{m}$, a subsequence $x^{\prime}$ of $x$ with length $w \leq m$ is a sequence of $w$ contiguous data points of $x$, i.e., $x^{\prime}=\left\{t_{p}, \ldots, t_{p+w-1}\right\}$, for $1 \leq p \leq m-w+1$.

Let $\langle X, Y\rangle$ be a time series dataset, where $X=\left\{x_{1}, \ldots, x_{n}\right\}$ is a set of $n$ time series instances and $Y=\left\{y_{1}, \ldots y_{n}\right\}$ models the time series labels (or classes), with $y_{i} \in L=\left\{l_{1}, \ldots, l_{g}\right\}$ being the set of $g$ classes. The shapelet discovery process in [39] consists of two steps: (i) identification of shapelet candidates, and (ii) shapelet evaluation.

In the first step the process extracts all the subsequences with length $w \in\left[w_{\text {min }}, w_{\text {max }}\right]$ for each time series $x_{i} \in X$, where $w_{\text {min }}$ and $w_{\max }$ are given. Thus, given a time series $x$ and the value $w$, the set $S_{x}^{w}=\left\{s_{j} \mid 1 \leq j \leq m-w+1\right\}$ is generated by sliding a window of size $w$ along $x$ and considering each possible subsequence. At the end of this step, we get the set of shapelet candidates $C=$ $\bigcup_{x \in X, w \in\left[w_{\min }, w_{\max }\right]} S_{x}^{w}$ derived from the input dataset $X$.

The second step evaluates the discriminatory power of each shapelet candidate $s \in C$ by estimating to which extent each shapelet $s$ is able to well separate time series in $X$ among the classes $L$. To this aim, given a candidate shapelet $s$, the method calculates the distances $D \in \mathbb{R}^{n}$ between $s$ and the $n$ time series $x_{i} \in X$. The distance between a time series and a subsequence is defined as:

Definition 3.3 (Distance). Given a distance function $d$, the distance between a time series $x$ of length $m$ and a subsequence $s$ of length $w \leq m$ is $\operatorname{dist}(x, s)=\min \left(d\left(s, x^{\prime}\right)\right) \forall x^{\prime} \in S_{x}^{w}$.

Intuitively, $\operatorname{dist}(x, s)$ is the distance between the subsequence $s$ and its best matching location along $x$. Therefore, given a shapelet $s$, the vector of distances is defined as $D=\{\operatorname{dist}(x, s) \mid \forall x \in X\}$. The most widely used distance function $d$ is the Euclidean distance ${ }^{1}$.

The method sorts the distances $D$ and finds an optimal split point to separate the instances in $X$ among the classes $L$ by maximizing the purity of each group with respect to the class value. The split

${ }^{1}$ We adopt the Euclidean distance on the normalized values. 
point is the value that maximizes the information gain [29]. If the entropy is used to measure the information gain, given a dataset $X$ with samples labeled with classes in the set $L$, then the entropy is $I(X)=-\sum_{l_{i} \in L} p\left(l_{i}\right) \log \left(p\left(l_{i}\right)\right)$, where $p\left(l_{i}\right)$ is the proportion of time series in class $l_{i}$. The information gain provides the difference of entropy between the dataset before and after the split. If there are two classes in the dataset and $X$ is split into $X_{1}$ and $X_{2}$, then the information gain is $I G\left(X,\left\langle X_{1}, X_{2}\right\rangle\right)=I(X)-\left(\left|X_{1}\right| /|X| \cdot I\left(X_{1}\right)+\right.$ $\left.\left|X_{2}\right| /|X| \cdot I\left(X_{2}\right)\right)$. Given the ordered distances $D$, the best split for a shapelet $s$ is found by scanning the distances and computing the information gain $I G$ for any split, corresponding to the middle point between two adjacent distances. Thus, a shapelet $s$ is a time series subsequence which separates the time series in $X$ among the classes in $L$ by maximizing the purity (measured by the information gain) with respect to each class.

Among the shapelets $s \in C$ the method in [39] returns the shapelet with the highest information gain while in [22] the best $h$ shapelets are selected and used for classification.

\section{METHODOLOGY}

The discriminatory power of shapelets and their simultaneously concise and informative format theoretically allows to represent instances of any type as distances from a set of shapelets. This representation enables to effectively classify unseen instances through any simple interpretable classifier such as decision trees, nearest neighbors and logistic regressors. that can be employed in Artificial Intelligence systems. The key contribution of our proposal is threefold. First, it allows us to define and discover shapelets for any type of data. Second, it simultaneously guarantees (i) an accuracy and running time comparable with the state-of-the-art, and (ii) high stability for the shapelets extracted. Third, it shows how to empower existing explanation methods with interpretable shapelet-based models. We target these goals by proposing DASH, an interpretable Data-Agnostic classification method via sHapelets. In the following, first we introduce shapelet definitions for other types of data, then we describe a procedure for effectively extracting them and use for building an interpretable classifier. Finally, we show how to integrate DASH in exiting explainers.

\subsection{Image, Tabular and Text Shapelets}

We extend here the notion of time series shapelets [39] to image, tabular and textual data. In the following we define how we adapt the shapelet definition to these data types. We underline that, independently from the type of data, given a dataset $\langle X, Y\rangle$, where $X=\left\{x_{1}, \ldots, x_{n}\right\}$ is a set of $n$ instances and $Y=\left\{y_{1}, \ldots y_{n}\right\}$ are the corresponding classes with $y_{i} \in L$, a shapelet $s$ is a sub-instance which separates the instances in $X$ among the classes $L$ by maximizing the purity of each group with respect to the class value.

4.1.1 Shapelet on Image Data. In a dataset of images an instance is modeled by a matrix of pixel values, where, like for time series, it is important to model and to capture the contiguity among the data points ${ }^{2}$.

${ }^{2}$ We restrict here to black and white images but all the definitions can be re-formulated for the RGB case.
Definition 4.1 (Image). An image $x$ is a $m \times q$ matrix where each element $x_{i, j} \in[0,255]$ represents a pixel value.

In this context, an image shapelet is a submatrix, i.e., an area of contiguous pixels, which well separates images of different classes. We define the notion of submatrix for representing an image shapelet as follows:

Definition 4.2 (Submatrix). Given an image $x$ of $m \times q$ pixels, a submatrix $x^{\prime}$ of $w \times v$ pixels with $w \leq m, v \leq q$, is a matrix of $w \times v$ contiguous pixels of $x$, i.e., a subset of contiguous rows and columns of the matrix $x$.

As for time series, the process of shapelet candidates identification generates the image submatrices starting from an image $x$ sliding a window of size $w \times v$ across $x$ and considering each derived submatrix. Also, the mechanism for the shapelet candidate evaluation is the same as that one used for the time series applying the distance introduced in Definition 3.3, considering the bi-dimensional nature of images.

4.1.2 Shapelet on Tabular Data. In a tabular (relational) dataset an instance consists in a record modeled as a set of valued features. More formally, we have:

Definition 4.3 (Record). A record $x \in \mathbb{R}^{m}$ is a set of $m$ attributevalue pairs $x=\left\{\left(a_{1}, v_{1}\right), \ldots,\left(a_{m}, v_{m}\right)\right\}$ where each $a_{i}$ is an attribute and $v_{i}$ is a value of the domain of $a_{i}$.

In this context, a record shapelet is a features subset formed by a subset of the features of the original dataset which well separates records of different classes. The main difference between record shapelets and time series or image shapelets derives from the fact that in tabular data there is no relation of contiguity (neither temporal or spatial) between the valued features. Hence, the process of shapelets discovery is based on the notion of features subsets.

Definition 4.4 (Feature Subset). Given a record $x$ of $m$ attributes, a feature subset $x^{\prime}$ with length $w \leq m$ is a set of $w$ attribute-value pairs such that $x^{\prime} \subseteq x$, and $\left|x^{\prime}\right|=w$.

We highlight that the definition of a record shapelet as a feature subset has a direct impact on the procedure of shapelet candidates identification (described in Section 3). Indeed, given an interval $\left[w_{\text {min }}, w_{\text {max }}\right]$, for every record $x \in X$ this process has to generate all the feature subsets of $x$ for every length $w \in\left[w_{\min }, w_{\max }\right]$. Also, the distance $\operatorname{dist}(x, s)$ between a shapelet candidate $s$ and a record $x$ must be computed considering the projection of the record with respect to the $w$ features contained in $s$. More formally,

Definition 4.5 (Feature Distance). Given a distance function $d$, the distance between a record $x$ and a feature subset $s$, formed by $w$ features $A$, is defined as $\operatorname{dist}(x, s)=d\left(\pi_{A}(x), s\right)$, where $\pi_{A}(x)$ is the projection function that given a record $x$ and a set of features $A$ returns only the feature-valued pairs of $x$ for the $w$ features in $A$.

4.1.3 Shapelet on Textual Data. In textual datasets, an instance is formed by the sequence of terms (tokens) contained in the text. The shapelet extraction process depends on the type of representation adopted to model the text instances. We represent a text instance using Tf-Idf [23], i.e., a vector where each feature value contains the term frequency factored by the inverse document frequency. 


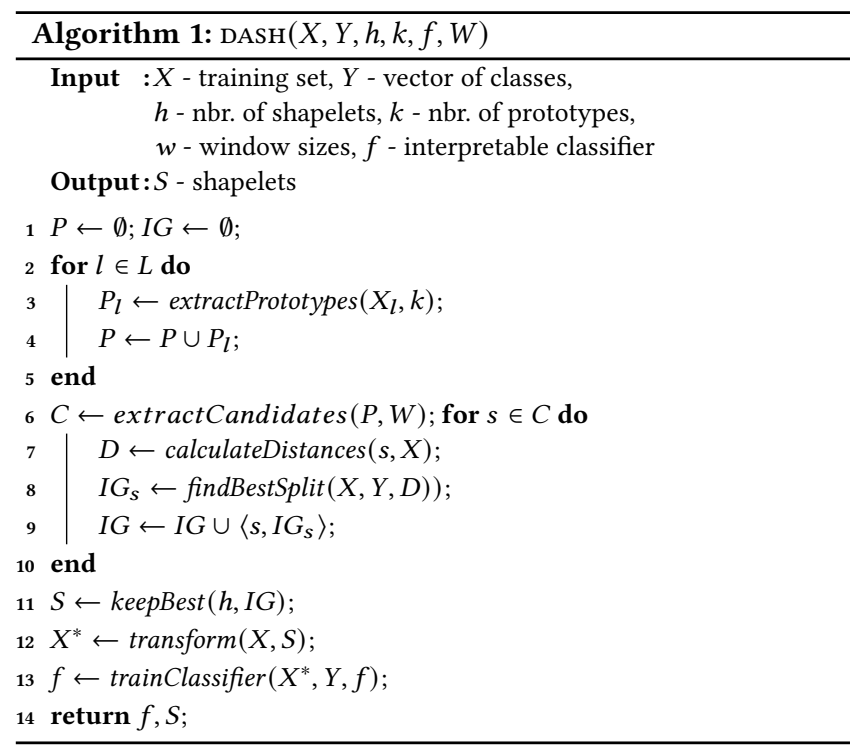

As a consequence, we get a data representation very similar to the tabular data, where we consider the terms as features and the Tf-Idf values associated with terms as values of the features. In turn, we can apply for textual data the same shapelet definition presented for tabular data.

We highlight that other text representations are possible. A direct alternative to Tf-Idf is the count based representation, where the value of each term simply contains the count/frequency of the term appearing in the text. The weakness of both the Tf-Idf and count/frequency representation is that we lose the order of the terms in the text. This drawback can be overcome by employing a word embedding representation [4]. Word embedding simultaneously allows us to retain the sequencing of terms in the text, and to compare texts with a distance function acting on the embeddings of the terms. We leave the definition of this kind of shapelet as future work and, in the rest of the paper, we assume the Tf-Idf representation for textual data.

\subsection{Prototype-Based Shapelet Discovery}

In this section we illustrate the algorithmic structure of DASH. The pseudo-code is reported in Algorithm 1. DASH first extracts some data prototypes representing the different classes (lines 2-5). Then, it performs a prototype-based shapelet discovery process exploiting the information gain (lines 6-12). Finally, DASH transforms the training set selecting the best shapelets, i.e., the most discriminatory ones, and trains an interpretable classifier on top of the transformed data (lines 13-14). Details are in the following.

4.2.1 Prototype-Based Candidate Shapelets. The first step of DASH consists in deriving a set of $k$ data prototypes $P_{l}$ for each class $l \in L$ in the training data $X$ (lines 2-5). To this end, DASH applies a prototype-based clustering, such as $k$-Means, or $k$-Medoid [35], to each group of training samples identified by the same class value. Thus, for each class $l$ the clustering result is the set $P_{l}$ composed by $k$ prototypes, i.e., $\left|P_{l}\right|=k$. We name $P=\bigcup_{l \in L} P_{l}$ the whole set of $k \times|L|$ prototypes.
The idea of using the centroids (or medoids), returned by a clustering algorithm, as data prototypes is based on the fact that every class is associated to a set of instances that may be generated by several distinct mechanisms and, in addition, some parts of an instance may describe and capture different behaviors and aspects [3]. In such a situation, the purpose of prototypes is to represent and summarize these sub-mechanisms in such a way that it becomes easier and faster to identify pieces of information characterizing groups of instances, i.e., the shapelets.

The rationale behind this choice is to simultaneously reduce the algorithm complexity and provide good and stable candidates across different applications of the algorithm. Indeed, as discussed in Section 2, a technique used for reducing the running time is the random selection of instances from the training set and the usage of this small set as candidate shapelets. However, even though this choice provides high performance with respect to the brute force algorithm [39], it does not ensure stability across different executions due to the random component. In the literature, it is shown that stability is a fundamental requirement for interpretability in order to gain user trust $[2,17]$. As we show in the experimental section, the randomness and the instability are markedly mitigated by DASH thanks to the usage of the prototype-based approach.

4.2.2 Shapelets Discovery. The second step of DASH consists in extracting the $h$ most informative shapelets from the prototypes $P$. To this aim, we exploit the algorithmic schema proposed in [39]. Given the prototypes $P$ and a set of window sizes $W$ (or alternatively an interval identified by $w_{\min }$ and $w_{\max }$ ), the set of shapelet candidates is built by extracting from the prototypes all the possible sub-instances with a window size $w \in W$ (line 6). In particular, the instances in $P$ are used for the shapelet candidates generation that, as explained above, depends on the type of data.

We recall that, since the contiguity property is not required for tabular and textual data, all the possible combinations of features should be considered for the candidate generation. To reduce the number of computations, and inspired by the pruning in [25], we define a heuristic for reducing the number candidates. Our strategy evaluates the information gain for feature subsets of growing length only if they are formed by feature subsets corresponding to shapelets in the top $h^{\prime} \gg h$ shapelets with highest information gain $^{3}$. This pruning principle is similar to that one applied by the Apriori algorithm [1] for the pattern generation, which exploits the anti-monotony property of the item set frequency. Note that, in our case there is no theoretical foundation on the fact that the anti-monotony property holds also for the information gain. The shapelet candidates $s \in C$ are evaluated on the training set $\langle X, Y\rangle$ by estimating their ability in separating the instances of $X$ in $|L|$ partitions $X_{1}, \ldots, X_{|L|}$, such that each partition either is pure or contains a high proportion of instances of a class (lines 7-11). The score $G_{s}$, computed for each shapelet candidate and provided by computing the information gain $I G\left(X,\left\langle X_{1}, \ldots, X_{|L|}\right\rangle\right)$, enables to select the top $h$ shapelets $S$ (line 12).

4.2.3 Data Transformation \& Classification. In order to train the classifier $f$ exploiting the shapelets $S$, DASH applies a transformation to the training data $X$. Let $h$ be the number of shapelets in $S$, each

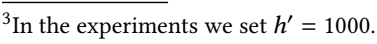




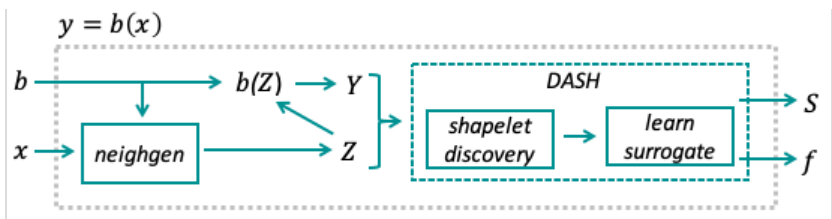

Figure 1: Workflow to extend local explanation methods.

instance $x \in X$ is mapped into a distance vector $x^{*} \in \mathbb{R}^{h}$. Each vector element $x_{i}^{*}$ contains the distance between $x$ and the $i^{t h}$ shapelet $s_{i} \in S$. We denote by $X^{*} \in \mathbb{R}^{n \times h}$ the transformed dataset (line 13). Thus, each instance of the training set is expressed as its distances from the representative information of the original data. Finally, these distance vectors $X^{*}$ are used to train the classifier $f$ (line 14). The data transformation allows separating the shapelet finding process from the learning of the classification model, enabling the use of any type of classifier.

4.2.4 Computational Complexity. The complexity mainly depends on the shapelet discovery and by the type of data. In case of time series, given $k \cdot|L|$ prototypes $P$, the total number of candidates of all possible lengths is: $\sum_{w \in W} \sum_{x \in P}\left(m_{x}-w+1\right)$. Hence, considering an average length of time series $\bar{m}$ the time complexity for the shapelet generation is $O\left(\bar{m}^{2} \cdot|P|\right)$. The computational cost of the evaluation of each shapelet candidate is $O(\bar{m} \cdot n)$, where $n=|X|$ because this step requires to compute the distance between the candidate and each instance in $X$. Thus, the overall time complexity of the shapelet discovery is $O\left(\bar{m}^{3} \cdot n \cdot|P|\right)$. This cost is lower than the complexity of the algorithm presented in [39] that is $O\left(\bar{m}^{3} \cdot n^{2}\right)$, due to the fact that they extract the shapelets from the whole training dataset $X$. In DASH we need to add the computational cost of $k$-means. However, the overall complexity is dominated by the shapelet extraction. In case of images, instances in $X$ are of size $m \times q$ and shapelets are bi-dimensional, so the number of candidates of all possible shapelet size $w \times v$ is: $\sum_{x \in P} \sum_{w \in W}\left(m_{x}-w+1\right) \sum_{v \in V}\left(q_{x}-v+\right.$ $1)$. Following the same reasoning used on time series we have that the cost of candidate generation is $O\left(\bar{m}^{2} \cdot \bar{q}^{2} \cdot|P|\right)$, while for candidate evaluation is $O(\bar{m} \cdot \bar{q} \cdot n)$. Hence, the overall cost without the prototype extraction, is $O\left(\bar{m}^{3} \cdot \bar{q}^{3} \cdot n \cdot|P|\right)$. In case of tabular and textual data, the process of shapelet candidate extraction has to generate all possible subset features. Without the pruning strategy, the only difference with respect to time series is that the number of possible candidates depends on the number of possible subsets of a record with $m$ attributes, i.e., $O\left(2^{m}\right)$. Considering the heuristic this number is lower and takes $O\left(\sum_{w \in W} w\left|S^{w-1}\right|\left|S^{1}\right|\right)$ as for Apriori.

\subsection{Shapelet-based Explanations}

In the eXplainable AI (XAI) we distinguish black box explanation from explanation by design [16]. In the first case, the idea is to couple an AI based on a black box model with an explanation method able to interpret the black box decisions. In the second case, the strategy is to substitute the obscure model with a transparent model in which the decision process is accessible by design. DASH classifiers belongs to this second family. However, in this section we show how they can be exploited also for the first case.
Table 1: Datasets description.

\begin{tabular}{|c|c|cccc|}
\hline type & name & training & test & dimensions & labels \\
\hline \multirow{5}{*}{ time series } & arrowhead & 36 & 175 & 251 & 3 \\
& ecg200 & 100 & 100 & 96 & 2 \\
& electric & 8926 & 7711 & 26 & 7 \\
& gunpoint & 50 & 150 & 150 & 2 \\
& italypower & 67 & 1029 & 24 & 2 \\
& phalanges & 1800 & 858 & 80 & 2 \\
\hline \multirow{5}{*}{ images } & cifar10 & $50 \mathrm{k}$ & $10 \mathrm{k}$ & $32 \times 32$ & 10 \\
& fashion & $60 \mathrm{k}$ & $10 \mathrm{k}$ & $28 \times 28$ & 10 \\
& mnist & $60 \mathrm{k}$ & $10 \mathrm{k}$ & $28 \times 28$ & 10 \\
\hline \multirow{5}{*}{ tabular } & ctg & 1488 & 638 & 35 & 10 \\
& diabetes & 537 & 231 & 8 & 2 \\
& ionoshpere & 245 & 106 & 34 & 2 \\
& parkinsons & 136 & 59 & 22 & 2 \\
& sonar & 145 & 63 & 60 & 2 \\
& vehicle & 592 & 254 & 18 & 4 \\
& wdbc & 398 & 171 & 18 & 4 \\
\hline \multirow{7}{*}{ text } & $20 n e w s$ & $11 \mathrm{k}$ & $7 \mathrm{k}$ & 1000 & 2 \\
& imdb & $25 \mathrm{k}$ & $25 \mathrm{k}$ & 1000 & 2 \\
\hline
\end{tabular}

The most used approach to explain AI black boxes is known as reverse engineering: the explanation is retrieved by observing what happens to the output, i.e., the AI decision, when changing the input in a controlled way. The explanation is generally extracted through a simple interpretable surrogate model that learns to locally or globally mime the behavior of the black box model. Global explanation consists in providing a way for interpreting any possible decision of a black box model ${ }^{4}$. On the other hand, local explanation consists in retrieving the reasons for the outcome returned by a black box model relatively to the decision for a specific instance [16]. Hence, a local interpretable surrogate model is used to approximate the AI behavior only in the "neighborhood" of the instance under analysis, i.e., with respect only to similar instances. LIME is a local explanation method [33] that randomly generates synthetic instances around the analyzed instance. Then it returns an explanation defined as a vector of features importance based on the coefficients of a linear regression model (a Lasso model) adopted as a local surrogate. The synthetic instances in the neighborhood are weighted according to their proximity to the instance of interest. The LORE explanation method [15] instead exploits a decision tree to return a local rule-based explanation that consists of a factual rule, revealing the reasons for the decision taken by the black box for the specific instance, but also a set of counterfactual rules, illustrating the AI logic used to get a different decision with minimum changes.

We propose to extend LIME and LORE following the workflow in Figure 1. The gray dotted box surrounds the shapelet-based explainer. It takes as input the black box classifier $b$ and the instance to explain $x$. First, it generates the neighborhood (neighgen) according to the procedures of LIME or LORE. Then, it labels the synthetic instances with the black box $Y=b(Z)$. Finally, a DAsH classifier is used as local shapelet-based interpretable surrogate model where the set of shapelets are used as explanation. We name shapelet-LIME

${ }^{4}$ We do not discuss it here for space reasons but also global explanation methods can be extended with DASH. 
(S-LIME) and shapelet-LORE (S-LORE) the methods LIME and LORE extended with DASH, respectively.

\section{EXPERIMENTS}

We experimented ${ }^{5}$ with six time series datasets, seven tabular datasets $^{6}$, three image datasets, and two text datasets. Table 1 reports the dataset details. All the datasets are open source and freely available. ${ }^{7}$ We turned the RGB images of cifar 10 into black and white images. In textual datasets, we removed the stopwords and punctuation, and we employed the Tf-Idf vectorization (with smoothed Idf) to represent the texts as vectors. We restricted to the 1000 terms with the highest frequency across the corpus. For 20news we only considered the two classes atheism and religion.

With respect to shapelet discovery on time series we compare DASH against the brute force algorithm ${ }^{8}$ BFA [39], the gradientdescent-based optimization algorithm ${ }^{9}$ GDBO [13], and the randomshapelet approach RND [32]. In addition, we extended RND to work on any type of data like DASH in order to be used as a baseline. We highlight that we do not compare against state-of-the-art classifiers for time series, images, tabular data and text because it is out from the purpose of this study ${ }^{10}$. Similarly, we do not compare against shapelet discovery algorithms which cannot be extended to extract shapelets on data different from time series.

We compare the performance in terms of running time (in seconds) and stability measured in terms of instability (the lower the better). The instability estimates the ability to provide similar shapelets for similar input instances. We define instability as follows. Given a dataset $X$, we extract various shapelet sets $\mathcal{S}=\left\{S_{1}, \ldots, S_{z}\right\}$. Then, we compare each shapelet set $S_{i}$ against any other set $S_{j} \in \mathcal{S}$ with $j \neq i$. For each shapelet $s \in S_{i}$, we find the most similar shapelet $s^{\prime} \in S_{j}$ and we compute the distance between $s$ and $s^{\prime}$ using the definitions previously illustrated. Thus, we model the instability in terms of the mean and standard deviation of these distances. For evaluating the interpretability of the shapelets we adopted the following classifiers, as implemented by the scikit-learn library: Decision Tree classifier (DT), k-Nearest Neighbor $(\mathrm{kNN})$ and Logistic Regression (LR). Moreover, we used the accuracy [35] to evaluate the predictive power of our shapelets. We selected appropriate parameters for the models to lower their complexity and to empower their interpretability [16]. In line with [5], for DTs, we limit the depth of the tree to $\max \left(4, \log _{2}(|L|)\right)$ because $(i)$ it is empirically shown that it is deep enough for being optimal and, (ii) a deeper tree would be more complex and less interpretable. Concerning $\mathrm{kNN}$, we adopt $1 \mathrm{NN}$ because, as humans, we are more comfortable in comparing one object against all the others rather than identifying

\footnotetext{
${ }^{5}$ Source code, datasets, and scripts for reproducing experiments are publicly available at https://github.com/riccotti/DASH.

${ }^{6}$ We restricted to datasets with continuous features but DASH also works on datasets with categorical features.

${ }^{7}$ Datasets https://www.cs.ucr.edu/ eamonn/time_series_data_2018/, http://yann.lecun com/exdb/mnist/, https://www.cs.toronto.edu/ kriz/cifar.html, https://www.kaggle com/zalando-research/, https://archive.ics.uci.edu/ml/datasets.php, http://qwone.com/ jason/20Newsgroups/, https://www.imdb.com/interfaces/.

${ }^{8}$ pyts library https://pyts.readthedocs.io/en/stable/generated/pyts.transformation. ShapeletTransform.html

${ }^{9}$ tslearn library https://tslearn.readthedocs.io/en/stable/gen_modules/shapelets/ tslearn.shapelets.ShapeletModel.html

${ }^{10}$ We discarded some state-of-the-art shapelet extractors and classifiers based on DNNs because either the source code is not available or they are not interpretable.
}

Table 2: Running time (in seconds) for shapelet discovery.

\begin{tabular}{|c|cccc|}
\hline dataset & DASH & GDBO & BFA & RND \\
\hline arrowhead & $5.271^{*}$ & 8.121 & 25.835 & $\underline{1.435}$ \\
ecg200 & $\underline{1.507}$ & 6.032 & 22.319 & $3.329^{*}$ \\
electric & $255.735^{*}$ & $\underline{85.784}$ & $>1 \mathrm{~h}$ & $>1 \mathrm{~h}$ \\
gunpoint & 7.177 & $5.543^{*}$ & 29.825 & $\underline{4.683}$ \\
italypower & $\underline{0.391}$ & 5.035 & 3.630 & $2.511^{*}$ \\
phalanges & $\underline{11.923}$ & $23.513^{*}$ & 3184.247 & 278.733 \\
\hline
\end{tabular}

Table 3: Shapelets instability as mean and standard deviation of distances across ten shapelet discovery runs.

\begin{tabular}{|c|cccc|}
\hline dataset & DASH & GDBO & BFA & RND \\
\hline ecg200 & $.014^{*} \pm .025$ & $.038 \pm .029$ & $\underline{.0 \pm .0}$ & $.285 \pm .233$ \\
gunpoint & $.006^{*} \pm .010$ & $.255 \pm .269$ & $\underline{.0 \pm .0}$ & $.114 \pm .175$ \\
italypower & $.033^{*} \pm .113$ & $.086 \pm .062$ & $\underline{.0 \pm .0}$ & $.249 \pm .273$ \\
phalanges & $.023^{*} \pm .055$ & $.183 \pm .182$ & $\underline{.0 \pm .0}$ & $.101 \pm .128$ \\
\hline
\end{tabular}

a set of most similar objects. For the LR, we used L2 regularization, and inverse regularization strength equals to one. To stress the fact that shapelets improve the interpretability, we compare the shapelet-based classifiers against the DF, $1 \mathrm{NN}$, and LR trained on the original datasets (not on the distances with the shapelets). We refer to them with the name NosH classifiers.

In the following, if not differently specified, we report results using these parameters for DASH and its competitors: number of top shapelets $h=10$, number of clusters $k=5$, window sizes $W=$ $\{2,4,6\}$ for italypower, $W=\{4,8,16\}$ for the others time series datasets, $W=\{(8,8),(16,16)\}$ for cifar $10, W=\{(7,7),(14,14)\}$ for fashion and mnist, $W=\{1,2,3,4\}$ for tabular datasets, and $W=\{1,2,3\}$ for textual datasets. As DASH prototypes we consider the centroids returned by k-Means [35].

In the Tables we underline the best performer and star* the second best performer. All the results were tested for their significance applying a Friedman test with Bonferroni-Dunn post-hoc evaluation [8]. The test was rejected for running time, instability and accuracy with p-values of $0.025,0.020,0.035$, respectively.

\subsection{Results on Time Series Data}

We qualitatively compare the interpretable classification of DASH with a NOSH classifier. We adopt a DT with maximum depth equals to two and the gunpoint dataset. On Figure $21^{\text {st }}$, we report the DASH-based DT showing that in case a given time series $x$ has a distance lower than 0.10 from the shapelet $s_{3}$ (in red), i.e., we can roughly say that $s_{3}$ is "contained" in $x$, then $x$ is labeled as Gun. This is what happens for the first instance in Figure $2\left(2^{\text {nd }}\right)$. Otherwise, if $s_{3}$ is not contained in $x$ and is closer than 0.01 to $s_{0}$ (in black) then $x$ is labeled as No Gun. This is what happens for the second instance $\left(3^{r d}\right)$. On Figure $24^{t h}$, we report the NOSH DT built on the original dataset with 150 features, i.e., a feature for each time value. The NOSH DT refers to these features indicating which values they must have. Unfortunately, if graphically illustrated along with the times series (right $5^{\text {th }}$ and $6^{\text {th }}$ ), these classifications are not immediately interpretable. Thus, the decision of NOSH classifiers, even in case 

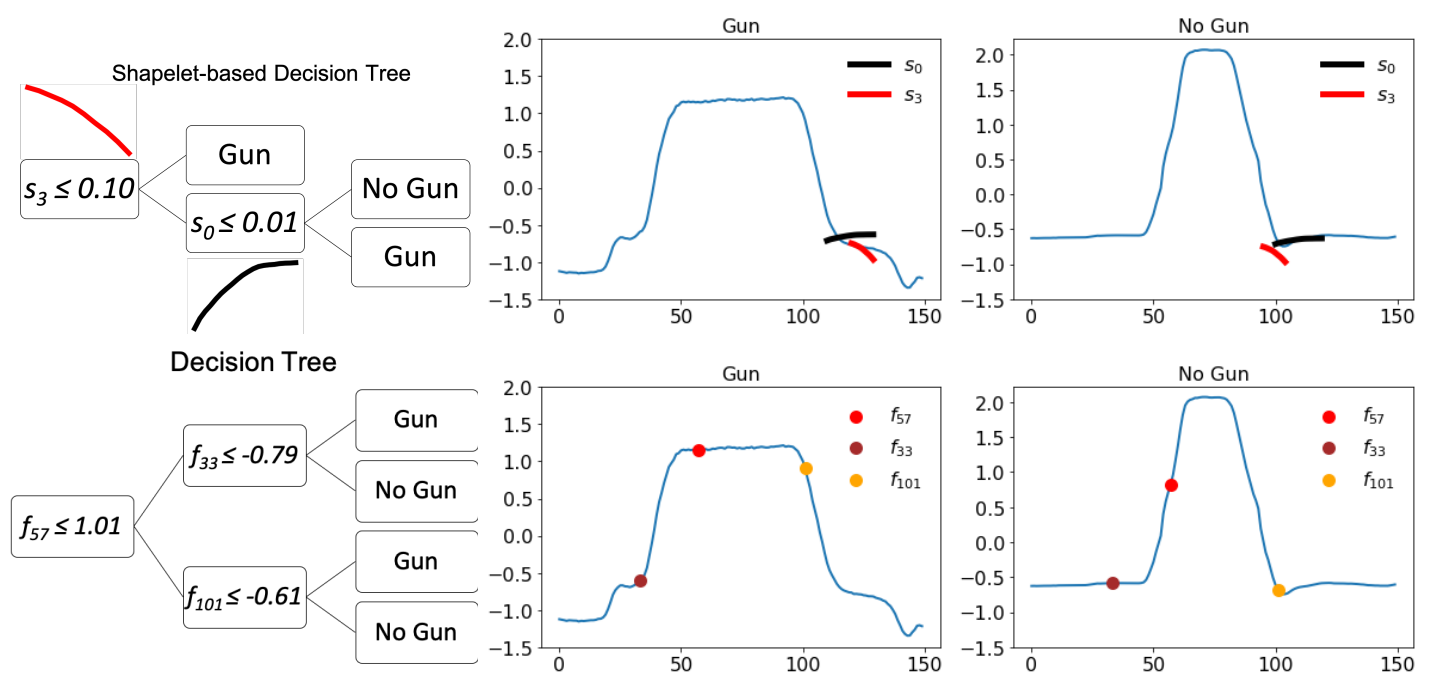

Figure 2: Interpretable classifications of two gunpoint instances using the DAsH-shapelet-based DT (top) and NOsH DT (bottom). Shapelets are shown at their best alignment.

Table 4: Accuracy for different interpretable classifiers and shapelets-based methods on time series.

\begin{tabular}{|c|c|c|c|c|c|c|c|c|c|c|c|c|c|c|c|}
\hline \multirow{2}{*}{ dataset } & \multicolumn{5}{|c|}{ DT } & \multicolumn{5}{|c|}{$1 \mathrm{NN}$} & \multicolumn{5}{|c|}{ LR } \\
\hline & DASH & GDBO & BFA & RND & NOSH & DASH & GDBO & BFA & RND & NOSH & DASH & GDBO & BFA & RND & $\mathrm{NOSH}$ \\
\hline arrowhead & $.583^{*}$ & .474 & .623 & .554 & .585 & $.446^{*}$ & .423 & .497 & .400 & .800 & $.600^{*}$ & .634 & .589 & .497 & .542 \\
\hline ecg200 & .800 & .640 & .740 & .780 & .804 & $.700^{*}$ & .560 & .750 & .720 & .880 & .770 & .640 & $.800^{*}$ & .800 & .729 \\
\hline electric & $.540^{*}$ & .548 & $\begin{array}{lll}- & -\end{array}$ & - & .484 & $.444^{*}$ & .479 & - & - & .549 & $.494^{*}$ & .513 & - & - & .229 \\
\hline gunpoint & $.807^{*}$ & .820 & .800 & .780 & .815 & .867 & .513 & $.793^{*}$ & .533 & .913 & .853 & .773 & $.847^{*}$ & .747 & .566 \\
\hline italypower & $.803^{*}$ & .641 & .812 & .769 & .951 & .813 & .662 & .550 & $.800^{*}$ & .955 & .791 & .561 & .764 & $.766^{*}$ & .741 \\
\hline phalanges & $.693^{*}$ & .640 & .705 & .683 & .693 & $.394^{*}$ & .519 & .387 & .614 & .761 & $.615^{*}$ & .613 & .638 & .609 & .547 \\
\hline
\end{tabular}

Table 5: Accuracy and running time for different interpretable classifiers and shapelets-based methods on images.

\begin{tabular}{|c|c|c|c|c|c|c|c|c|c|c|c|c|}
\hline \multirow{2}{*}{ dataset } & \multirow{2}{*}{ classes } & \multicolumn{3}{|c|}{ DT } & \multicolumn{3}{|c|}{$1 \mathrm{NN}$} & \multicolumn{3}{|c|}{ LR } & \multicolumn{2}{|c|}{ Time } \\
\hline & & DASH & RND & $\mathrm{NOSH}$ & DASH & RND & NOSH & DASH & RND & NOSH & DASH & RND \\
\hline \multirow{3}{*}{ cifar 10} & $\overline{\text { all }}$ & $.180^{*}$ & .171 & .218 & $.153^{*}$ & .149 & .303 & .212 & $.206^{*}$ & .131 & 154.482 & 2495.121 \\
\hline & $0,1,2,3$ & .445 & $.443^{*}$ & .425 & .305 & .296 &.$\overline{301^{*}}$ &.$\overline{405^{*}}$ & .435 & .401 & 59.327 & 217.718 \\
\hline & 0,1 & .762 & .733 & $.745^{*}$ & .695 & .557 & $.607^{*}$ & .742 &.$\overline{735^{*}}$ & .705 & 1.169 & 47.412 \\
\hline \multirow{3}{*}{ fashion } & all & $\overline{.511}$ & .580 & .645 & $\overline{.368}$ & .333 & .850 & .744 & .732 & .461 & 162.668 & 1684.277 \\
\hline & $0,1,2,3$ & .721 & $.751^{*}$ & .873 & .771 & .611 & $.711^{*}$ & $.847^{*}$ & .791 & .927 & 27.704 & 276.358 \\
\hline & 0,1 & $.942^{*}$ & .924 & .978 & $.833^{*}$ & .805 & .939 & $.941^{*}$ & .866 & .984 & 9.983 & 57.012 \\
\hline \multirow{3}{*}{ mnist } & all & .431 & .378 & .596 & .336 & .287 & .969 & .788 & .709 & .425 & 176.684 & 156.023 \\
\hline & $0,1,2,3$ & $.710^{*}$ & .703 & .911 & $.623^{*}$ & .514 & $\overline{.743}$ & $\overline{.796^{*}}$ & .761 & .978 & 27.483 & $\overline{311.008}$ \\
\hline & 0,1 & .991 & .910 & $\overline{.996}$ & .962 & .828 & $\overline{.978}$ & .990 & .910 & $\overline{.999}$ & $\overline{18.486}$ & 68.276 \\
\hline
\end{tabular}

of higher accuracy, is not entirely interpretable and not entirely acceptable as based on minimum information, i.e., time points, not easy to exploit in human reasoning.

Table 2 reports the running time. Except for gunpoint, DASH is always the first or second best performer with a time markedly lower than BFA and comparable with GDBO and RND. In Table 3 we illustrate the instability for ten runs of the shapelet discovery methods. As expected, the BFA is perfectly stable as it always returns the same set of shapelets $S$. DASH is the second best performer, and its instability is one order of magnitude lower than those of GDBO and RND. Table 4 reports the accuracy ${ }^{11}$. To have a fair evaluation, the best performers are considered only among the shapelet-based classifiers. This choice is also justified by the previous observations relating to the inadequacy of NOsH classifiers employed for interpretable time series classification. When a DT is used, DASH generally leads to the second best classification performance, GDBO

${ }^{11}$ Missing values "-" in Table 4 refers to running time of shapelet discovery processes higher than one hour and not reported. 


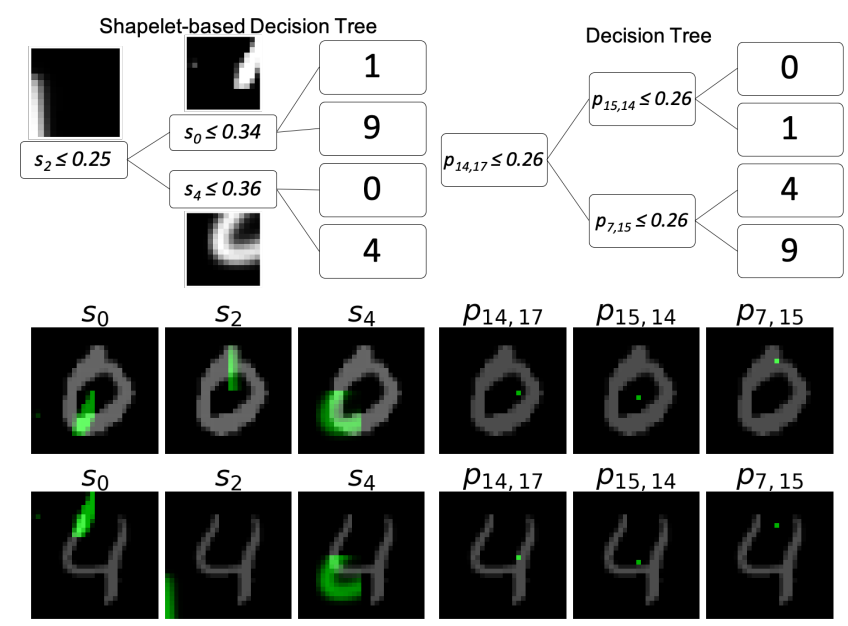

Figure 3: Interpretable classifications of four instances of mnist using the DASH-shapelet-based DT (left) and NOSH DT (right). Shapelets are shown at their best alignment.

and BFA to the best performance depending on the dataset, while RND is always the worst performer. Thus, DASH shapelets adopted by DT lead to high accuracy in various situations. This result is particularly relevant because, among the three classifiers, the DT, is perhaps the most interpretable one because it does not only allow direct reasoning and decomposition into logical rules, but also to infer how to revert the classification, i.e., counterfactual reasoning $[6,15]$. When $1 \mathrm{NN}$ or LR are used as classifiers DASH shapelets lead to the best performance for gunpoint and italypower. The most remarkable result from this Table is that DASH, independently form the dataset or interpretable classifier, guarantees very high accuracy. Hence, neither the dataset nor the type of classifier negatively influences the performance.

\subsection{Results on Image Data}

Similarly to the previous section, we first report a qualitative example on mnist using a DT with maximum depth equals to two and considered only instances with labels in $\{0,1,4,9\}$. Figure 3 shows the tree structures and the classification of two instances using the DASH DT on the left, and the NOSH DT on the right. The DASH-based DT shows that, if a given image $x$ "does not contain" $s_{2}$, i.e., it has not a submatrix black and with a blurred straight white line, then it can represent a " 0 " or a " 4 ". If $s_{4}$ is contained, i.e., it has a sort of a quarter-circle part, then it is a "0", otherwise it is a " 4 ". Similar reasoning can be done for the other classes. On the other hand (Figure 3 right), the NOsH DT focuses on the value of three pixels to return a classification. As humans, we cannot fully accept this kind of decision because it is far away from the logical process that we follow in recognizing the different digits. Indeed, as humans we reason on parts of the image instead of individual pixels. Thus, even if the accuracy of NOSH classifiers can be higher than shapelet-based classifiers (see Table 5), to have a fully interpretable process we should opt for the second ones.

Table 5 reports the classification accuracy and the running time for the image datasets. To observe the discriminatory power of

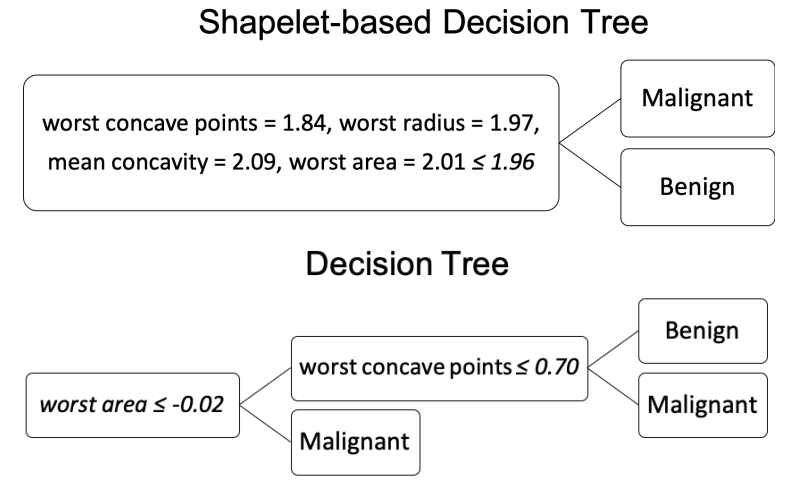

Figure 4: DASH DT, and NOSH DT for wdbc.

the top $h=10$ shapelets extracted, we performed the experiments considering different sets of classes: all the ten classes, $\{0,1,2,3\}$, and $\{0,1\}$. Indeed, a higher number of classes requires a higher number of shapelets for having good performances. Thus, setting $h=10$ allows us to observe the goodness of the few shapelets extracted. In 33 experiments out of 36, DASH is always one of the two best classifiers in terms of accuracy. DASH is also at least five times faster than RND typically having a running time always lower than its shapelet-based competitors.

\subsection{Results on Tabular Data}

Figure 4 compares the DASH and NOSH DTs for on wdbc. The first DT has a unique split involving a record shapelet composed of four feature-valued pairs. If the distance between an instance and this record shapelet is lower than 1.96, then the class is "Maglignant" otherwise is "Benign". We can summarize the interpretability as, if a record $x$ is similar to these attributes, then the classification is "Maglignant". The record-shapelet-based DT resembles oblique trees [27]. On the other hand, NOSH DT only refers to two features. Both decision trees have an accuracy of 0.94 . Table 6 reports the accuracy and the running time for tabular datasets. The higher expressiveness of the shapelet-records makes DASH to be the best classifier when DT and LR is used. On the other hand, NOsH has the highest accuracy for $1 \mathrm{NN}$. Due to the fact that various features subsets must be tested as candidates, the running time of DASH is not markedly lower than $\mathrm{RND}^{12}$.

\subsection{Results on Text Data}

In Figure 5 we compare DASH and NOSH for 20news. Similarly to tabular data, DASH DT presents two conditions modeled by term shapelets. The similarity/dissimilarity to a particular set of terms is not a complex condition to understand, and we can easily find some of these terms in the textual data to confirm the intuition. On the other hand, the NOsH DT has a single term for each split. In this case, the simplicity of the classifier can be viewed as an inadequate or poor classifier. Table 7 shows the accuracy and the running time for text datasets: DASH is clearly superior to RND. Indeed, RND did not terminate within an hour, and the running time

\footnotetext{
${ }^{12}$ For DASH and RND we exploit the heuristic pruning strategy described in Section 4.
} 
Table 6: Accuracy and running time for different interpretable classifiers and shapelets-based methods on tabular data.

\begin{tabular}{|c|c|c|c|c|c|c|c|c|c|c|c|}
\hline \multirow{2}{*}{ dataset } & \multicolumn{3}{|c|}{ DT } & \multicolumn{3}{|c|}{$1 \mathrm{NN}$} & \multicolumn{3}{|c|}{ LR } & \multicolumn{2}{|c|}{ Time } \\
\hline & DASH & RND & NOSH & DASH & RND & $\mathrm{NOSH}$ & DASH & RND & NOSH & DASH & RND \\
\hline $\operatorname{ctg}$ & .821 & .811 & .821 & $.779^{*}$ & .811 & .996 & .821 & .811 & $.730^{*}$ & 52.671 & 1296.583 \\
\hline diabetes & .771 & $.753^{*}$ & .730 & .784 & $.740^{*}$ & .690 & .788 & $.775^{*}$ & .611 & 3.200 & 11.802 \\
\hline ionoshpere & .934 & .877 & $.890^{*}$ & .915 & .755 & $.858^{*}$ & .953 & $.915^{*}$ & .626 & $\underline{26.609}$ & 26.640 \\
\hline parkinsons & .881 & .831 & $.854^{*}$ & $.746^{*}$ & $.746^{*}$ & .939 & .851 & $.847^{*}$ & .746 & $\underline{16.688}$ & 19.148 \\
\hline sonar & .730 & .587 & $.708^{*}$ & $.667^{*}$ & $.667^{*}$ & .871 & .746 & $.571^{*}$ & .548 & 29.843 & 27.823 \\
\hline vehicle & $\overline{.650}$ & $.654^{*}$ & .672 & $.449^{*}$ & .425 &.$\overline{693}$ & .652 & .634 & $.346^{*}$ & $\underline{72.898}$ & $\overline{95.969}$ \\
\hline$w d b c$ & .942 & .915 & $.936^{*}$ & $.942^{*}$ & .930 & .956 & .971 & $.971^{*}$ & .773 & 33.383 & 114.398 \\
\hline
\end{tabular}

Table 7: Accuracy and running time for different interpretable classifiers and shapelets-based methods on text.

\begin{tabular}{|c|ccc|ccc|ccc||cc|}
\hline \multirow{2}{*}{ dataset } & \multicolumn{3}{|c|}{ DT } & \multicolumn{3}{c|}{ 1NN } & \multicolumn{3}{c||}{ LR } & \multicolumn{3}{c|}{ Time } \\
& DASH & RND & NOSH & DASH & RND & NOSH & DASH & RND & NOSH & DASH & RND \\
\hline 20news & $\underline{.596}$ & .519 & $\underline{.596}$ & $\underline{.532}$ & .519 & $.531^{*}$ & $\underline{.598}$ & $.504^{*}$ & .499 & $\underline{\underline{175.062}}$ & 663.239 \\
imdb & $.655^{*}$ & - & $\underline{.676}$ & $\underline{.598}$ & - & $.539^{*}$ & $\underline{.655}$ & - & .525 & $\underline{1272.635}$ & $>1 \mathrm{~h}$ \\
\hline
\end{tabular}

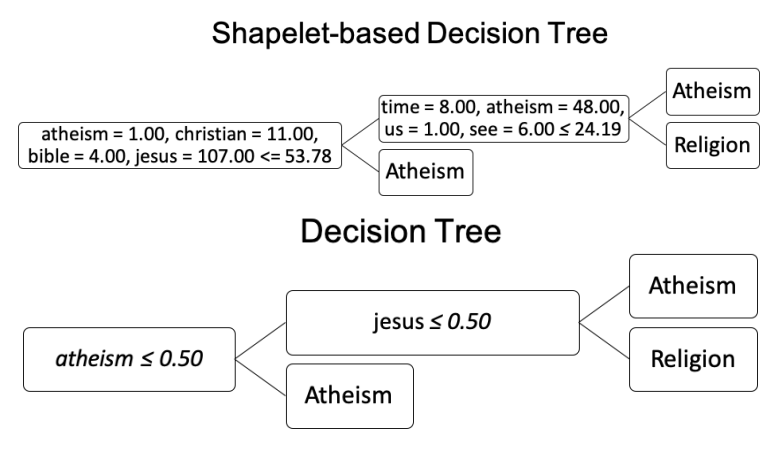

Figure 5: DASH DT, and NOSH DT for 20news.

of DASH is definitively lower than RND. In addition, the accuracy of DAsH classifiers is higher than the others nearly in all cases.

\subsection{Impact of Parameters}

We investigate the effect of varying the parameters of DASH: the number of shapelets $h$ and clusters $k$. Figure 6 reports these results for DT on gunpoint, mnist, wdbc, 20news. We notice that the number of shapelets $h$ mainly affects the accuracy while the number of clusters $k$ the running time. In particular, a low number of shapelets leads to poor performance (an accuracy lower than 0.8), while $h>10$ does not lead to particular improvements for datasets with two classes. Instead, for the mnist dataset, we observe that there is a clear improvement when using more than ten shapelets. We also notice that a too high number of shapelets decreases the accuracy of the 20news dataset. The number of clusters does not impact very much on the accuracy, but using only $k=1$ cluster per class (blue line), i.e., one prototype, always has the worst performance. On the other hand, the running time linearly increases with $k$.
Table 8: Average fidelity of standard explanation methods vs. explanation methods extended with DASH on time series.

\begin{tabular}{|c|cc|cc|}
\hline dataset & LIME & S-LIME & LORE & S-LORE \\
\hline ecg200 & .9634 & .9345 & .9996 & .9953 \\
gunpoint & .9412 & .9886 & .9998 & .9977 \\
italypower & .9793 & .9126 & .9812 & .9568 \\
phalanges & .9893 & .9435 & .9901 & .9735 \\
\hline
\end{tabular}

\subsection{Impact on Explanation Methods}

We analyze the impact of using DASH for extending LIME and LORE. We show the performance of s-LIME and s-LORE in terms of fidelity and we provide an insight on the quality of the explanations and the gain of comprehensibility. In line with [15], we measure the fidelity as the accuracy obtained by comparing the labels of the interpretable local model with the labels assigned by the black box classifier being explained. In this experiment we explain an AI system adopting a Convolutional Neural Network [11] (CNN) as black box. We trained CNNs for the datasets ecg200, gunpoint, italypower and phalanges. As validation set we used $25 \%$ of the training set ${ }^{13}$. We provided to the local explainers knowledge about the distributions of the training set and we explained 100 randomly selected instances from the test set of each dataset.

We compared the original version of LIME and LORE with the DASH based versions. Table 8 shows that in terms of average fidelity the two alternatives are comparable and that both achieve very high performance. This result is also confirmed by the $\mathrm{Ne}-$ menyi statistical test. Figure 8 shows the Critical Difference (CD) diagrams [8]. Two methods are tied if the null hypothesis that their performance is the same cannot be rejected using the Nemenyi test at $\alpha=0.05$. The two diagrams show that there is no statistically significant difference in terms of performance between the pairs of explainers. Figure 7 highlights that in terms of comprehensibility the explanations provided by S-LIME and S-LORE are more effective

\footnotetext{
${ }^{13}$ Details about the CNN can be found int he code repository.
} 


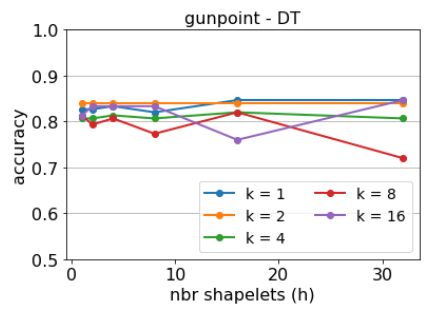

gunpoint

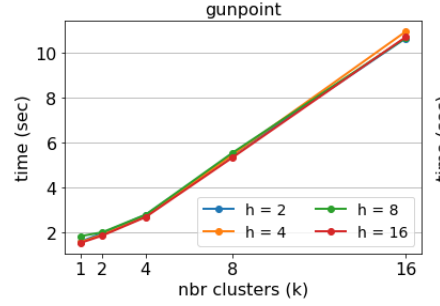

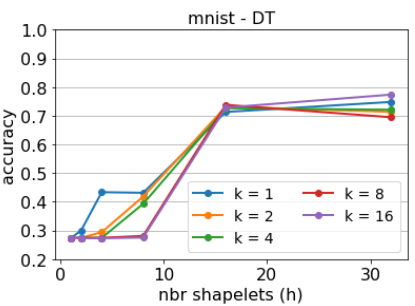

mnist

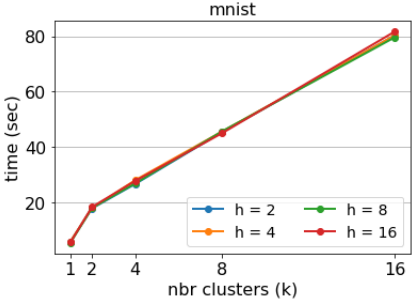

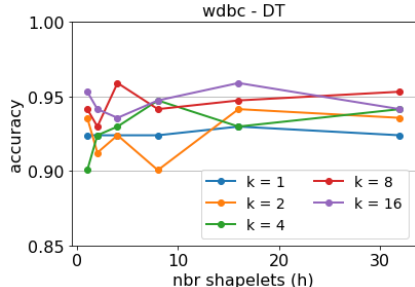

$w d b c$

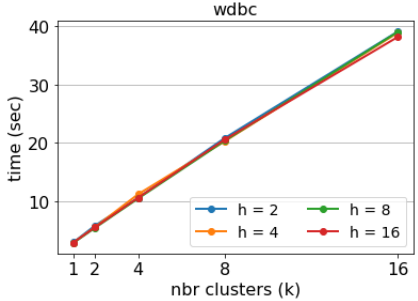

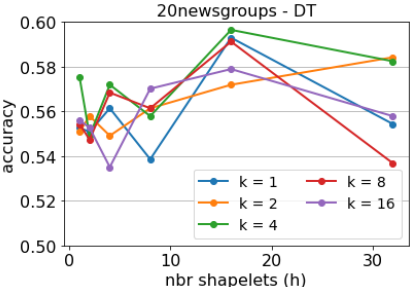

20newsgroups

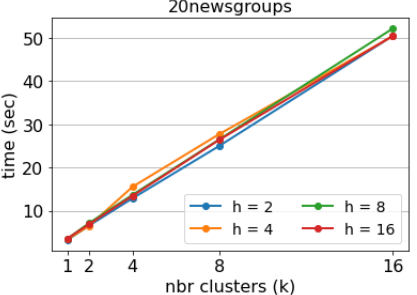

Figure 6: Accuracy and running time varying the number of shapelets $h$ and number of clusters $k$. on time series datasets for DT on gunpoint, mnist, wdbc, 20news (similar results for other experiments).
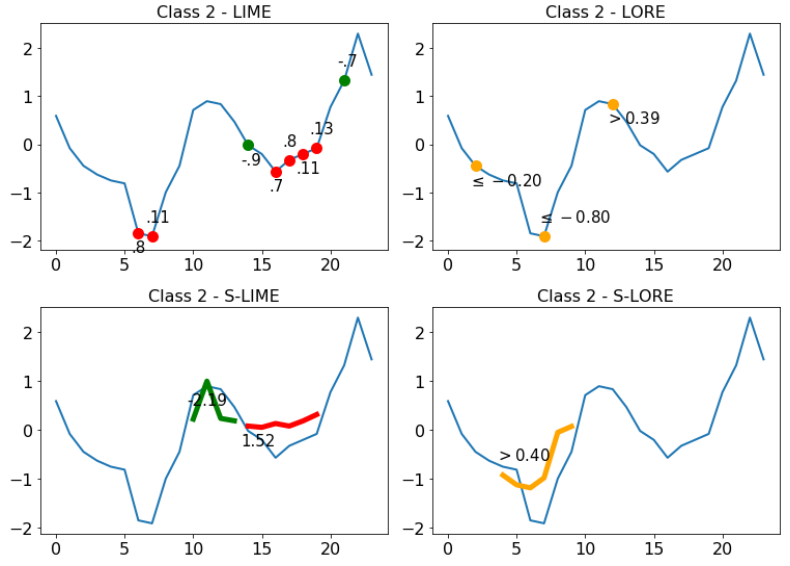

Figure 7: Explanations on italypower. LIME/s-LIME: green indicates that the point/shapelet pushes towards the class, red against it. LORE/s-LORE: for every point/shapelet is shown the condition responsible for the classification.

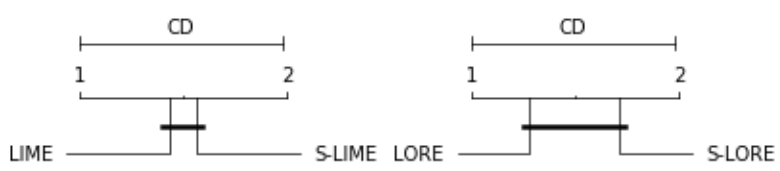

Figure 8: CD for fidelity with Nemenyi test at $\alpha=0.05$.

and intuitive: while LIME and LORE provide explanations focused on single points of the time series classification, their extended versions explain the classification by using trends and patterns of the time series. We do not report experiments using tabular data, images and text due to space reasons but such explanations can be easily extracted with the proposed method.

\section{CONCLUSION}

We have proposed DASH an interpretable data-agnostic classification approach based on shapelets defined on time series, images, textual and tabular data. DASH exploits data prototypes obtained by a clustering process to speed-up and stabilize the shapelet computation. Although DASH is an explainable by design AI approach, we show how it can be employed to mime and explain an AI decision system based on an obscure machine learning model. A wide experimentation shows that the classifications obtained with DASH are effective, interpretable and the decision process based on human-understandable parts can be easily accepted from humans. Moreover, local explanation methods extended with DASH achieve an evident gain in comprehensibility while having comparable fidelity to the state-of-art explainers. Several research directions can be mentioned as future work for recovering DAsH limitations. First, alternative techniques based on input data approximation can be explored for markedly speeding up the whole algorithm. Moreover, we would like to extend DASH for the classification of alternative types of data like item sequences, mobility trajectories, genomics sequences, etc. Finally, we imagine to exploit DASH for explaining complex black box classifiers such as siamese neural networks.

\section{ACKNOWLEDGMENTS}

This work has been partially supported by the European Community H 2020 programme under the funding schemes: G.A. 871042 SoBigData++, G.A. 952026 HumanE AI Net, G.A. 825619 AI4EU, and G.A. 952215 TAILOR. This work has been partially supported by University of Pisa, Progetti di Ricerca di Ateneo 2018-43 on Emerging Trends in Data Science.

\section{REFERENCES}

[1] Rakesh Agrawal and Ramakrishnan Srikant. 1994. Fast Algorithms for Mining Association Rules in Large Databases. In VLDB. Morgan Kaufmann, 487-499.

[2] David Alvarez-Melis and Tommi S. Jaakkola. 2018. Towards Robust Interpretability with Self-Explaining Neural Networks. In NeurIPS. 7786-7795.

[3] Timothy L Bailey, Charles Elkan, et al. 1994. Fitting a mixture model by expectation maximization to discover motifs in bipolymers. 
[4] Yoshua Bengio, Réjean Ducharme, Pascal Vincent, and Christian Janvin. 2003. A Neural Probabilistic Language Model. 7. Mach. Learn. Res. 3 (2003), 1137-1155.

[5] Dimitris Bertsimas and Jack Dunn. 2017. Optimal classification trees. Mach. Learn. 106, 7 (2017), 1039-1082.

[6] Ruth M. J. Byrne. 2019. Counterfactuals in Explainable Artificial Intelligence (XAI): Evidence from Human Reasoning. In IFCAI. ijcai.org, 6276-6282.

[7] Mark W. Craven and Jude W. Shavlik. 1995. Extracting Tree-Structured Representations of Trained Networks. In NIPS. MIT Press, 24-30.

[8] Janez Demsar. 2006. Statistical Comparisons of Classifiers over Multiple Data Sets. 7. Mach. Learn. Res. 7 (2006), 1-30.

[9] Huiqi Deng, Weifu Chen, Andy Jinhua Ma, Qi Shen, Pong C. Yuen, and Guocan Feng. 2018. Robust Shapelets Learning: Transform-Invariant Prototypes. In PRCV (3) (Lecture Notes in Computer Science, Vol. 11258). Springer, 491-502.

[10] Finale Doshi-Velez and Been Kim. 2017. Towards a rigorous science of interpretable machine learning. arXiv preprint arXiv:1702.08608 (2017).

[11] Hassan Ismail Fawaz, Germain Forestier, Jonathan Weber, Lhassane Idoumghar and Pierre-Alain Muller. 2019. Deep learning for time series classification: a review. Data Min. Knowl. Discov. 33, 4 (2019), 917-963.

[12] Daniel Gordon, Danny Hendler, and Lior Rokach. 2012. Fast Randomized Model Generation for Shapelet-Based Time Series Classification. CoRR abs/1209.5038 (2012).

[13] Josif Grabocka, Nicolas Schilling, Martin Wistuba, and Lars Schmidt-Thieme. 2014. Learning time-series shapelets. In KDD. ACM, 392-401.

[14] Josif Grabocka, Martin Wistuba, and Lars Schmidt-Thieme. 2015. Scalable Dis covery of Time-Series Shapelets. CoRR abs/1503.03238 (2015). arXiv:1503.03238 http://arxiv.org/abs/1503.03238

[15] Riccardo Guidotti, Anna Monreale, Fosca Giannotti, Dino Pedreschi, Salvatore Ruggieri, and Franco Turini. 2019. Factual and Counterfactual Explanations for Black Box Decision Making. IEEE Intell. Syst. 34, 6 (2019), 14-23.

[16] Riccardo Guidotti, Anna Monreale, Salvatore Ruggieri, Franco Turini, Fosca Giannotti, and Dino Pedreschi. 2019. A Survey of Methods for Explaining Black Box Models. ACM Comput. Surv. 51, 5 (2019), 93:1-93:42.

[17] Riccardo Guidotti and Salvatore Ruggieri. 2019. On The Stability of Interpretable Models. In IFCNN. IEEE, 1-8.

[18] Jon Hills, Jason Lines, Edgaras Baranauskas, James Mapp, and Anthony J. Bagnall 2014. Classification of time series by shapelet transformation. Data Min. Knowl. Discov. 28, 4 (2014), 851-881.

[19] Cun Ji, Chao Zhao, Shijun Liu, Chenglei Yang, Li Pan, Lei Wu, and Xiangxu Meng 2019. A fast shapelet selection algorithm for time series classification. Comput Networks 148 (2019), 231-240.

[20] Isak Karlsson, Panagiotis Papapetrou, and Henrik Boström. 2016. Generalized random shapelet forests. Data Min. Knowl. Discov. 30, 5 (2016), 1053-1085.

[21] Jessica Lin, Eamonn J. Keogh, Li Wei, and Stefano Lonardi. 2007. Experiencing SAX: a novel symbolic representation of time series. Data Min. Knowl. Discov. 15,
2 (2007), 107-144

[22] Jason Lines, Luke M. Davis, Jon Hills, and Anthony J. Bagnall. 2012. A shapelet transform for time series classification. In KDD. ACM, 289-297.

[23] Christopher D. Manning, Prabhakar Raghavan, and Hinrich Schütze. 2008. Introduction to information retrieval. Cambridge University Press.

[24] Tim Miller. 2019. Explanation in artificial intelligence: Insights from the social sciences. Artif. Intell. 267 (2019), 1-38.

[25] Abdullah Mueen, Eamonn J. Keogh, and Neal E. Young. 2011. Logical-shapelets: an expressive primitive for time series classification. In KDD. ACM, 1154-1162.

[26] Bonnie M. Muir. 1987. Trust Between Humans and Machines, and the Design of Decision Aids. Int. 7. Man Mach. Stud. 27, 5-6 (1987), 527-539.

[27] Sreerama K. Murthy, Simon Kasif, and Steven Salzberg. 1994. A System for Induction of Oblique Decision Trees. F. Artif. Intell. Res. 2 (1994), 1-32.

28] Frank Pasquale. 2015. The black box society. Harvard University Press.

[29] J Ross Quinlan. 1993. C4. 5: Programs for Machine Learning. Elsevier.

[30] Thanawin Rakthanmanon and Eamonn Keogh. 2013. Fast shapelets: A scalable algorithm for discovering time series shapelets. In ICDM. SIAM, 668-676.

[31] Alexandre Refregier. 2003. Shapelets: I. A Method for Image Analysis. Monthly Notices of the Royal Astronomical Society 338 (06 2003).

[32] Xavier Renard, Maria Rifqi, Walid Erray, and Marcin Detyniecki. 2015. Randomshapelet: An algorithm for fast shapelet discovery. In 2015 IEEE International Conference on Data Science and Advanced Analytics, DSAA 2015, Campus des Cordeliers, Paris, France, October 19-21, 2015. IEEE, 1-10.

[33] Marco Túlio Ribeiro, Sameer Singh, and Carlos Guestrin. 2016. "Why Should I Trust You?": Explaining the Predictions of Any Classifier. In KDD. ACM, 11351144.

[34] Payam Sabzmeydani and Greg Mori. 2007. Detecting Pedestrians by Learning Shapelet Features. In CVPR. IEEE Computer Society.

[35] Pang-Ning Tan, Michael Steinbach, and Vipin Kumar. 2016. Introduction to data mining. Pearson Education India.

[36] Yichang Wang, Rémi Emonet, Élisa Fromont, Simon Malinowski, Etienne Menager, Loïc Mosser, and Romain Tavenard. 2019. Learning Interpretable Shapelets for Time Series Classification through Adversarial Regularization. CoRR abs/1906.00917 (2019). arXiv:1906.00917 http://arxiv.org/abs/1906.00917

[37] Martin Wistuba, Josif Grabocka, and Lars Schmidt-Thieme. 2015. Ultra-Fast Shapelets for Time Series Classification. CoRR abs/1503.05018 (2015).

[38] W. Yao and Z. Deng. 2012. A robust pedestrian detection approach based on shapelet feature and Haar detector ensembles. Tsinghua Science and Technology 17, 1 (2012), 40-50.

[39] Lexiang Ye and Eamonn J. Keogh. 2009. Time series shapelets: a new primitive for data mining. In KDD. ACM, 947-956.

[40] Lexiang Ye and Eamonn J. Keogh. 2011. Time series shapelets: a novel technique that allows accurate, interpretable and fast classification. Data Min. Knowl. Discov. $22,1-2$ (2011), 149-182. 